\title{
Calidad del fruto y pérdidas poscosecha de banano orgánico (Musa acuminata) en el Ecuador
}

\section{(Fruit Quality and Post-Harvest Losses of Organic Bananas (Musa acuminata) in Ecuador)}

\author{
Wilson Vásquez-Castillo ${ }^{1}$, Mauricio Racines-Oliva ${ }^{1}$, Pablo Moncayo ${ }^{1}$, \\ William Viera ${ }^{2}$ y María Seraquive ${ }^{1}$
}

\begin{abstract}
Resumen
Ecuador es el primer exportador de banano del mundo con más de 317 millones de cajas por año, lo que aporta el 26 $\%$ del PIB agrícola y genera alrededor de 2.5 millones de empleos directos e indirectos. Ecuador produce 19000 ha y exporta 36000 t de banano orgánico. Respecto a las pérdidas poscosecha, estas fluctúan entre el 10 y 80 \% y son causadas por un inadecuado manejo tanto de la poscosecha como en lo agronómico. Este estudio tiene el objetivo de determinar la calidad física y química de la fruta orgánica y cuantificar las pérdidas poscosecha. La investigación se llevó a cabo en una plantación de banano orgánico ubicada en la provincia de Los Ríos (Ecuador). Se utilizó un diseño de bloques completamente al azar (DBCA) en arreglo factorial $7 \times 2$. Los factores fueron las épocas de cosecha (febrero-agosto), y la calidad de la fruta de exportación (primera y segunda) con 3 repeticiones. Las variables estudiadas se determinaron con base en 20 racimos. De los resultados obtenidos, se deduce que la calidad física y química del fruto está influenciada por la época de cosecha, ya que los racimos crecieron y desarrollaron en diferentes condiciones ambientales. En general, el 82 \% de la producción se destina para la exportación, debido a que cumple los estándares de calidad.
\end{abstract}

\section{Palabras clave}

Banano orgánico, calidad física, calidad química, pérdidas poscosecha, descartes de la fruta.

\begin{abstract}
Ecuador is the world's first banana exporter with more than 317 million boxes per year, and contributes $26 \%$ of agricultural GDP, generating around 2.5 million direct and indirect jobs. Ecuador produces 19000 ha and exports 36000 t organic bananas. Postharvest losses fluctuate between 10 and $80 \%$, caused by inadequate postharvest and crop management. The present study aims to determine the physical and chemical quality of organic fruit and quantify post-harvest losses. The research was carried out in the organic banana plantation located in the province of Los Ríos (Ecuador). A completely randomized block design (DBCA) was used in a $7 \times 2$ factorial arrangement. The factors were: 1) harvest time (February-August), and 2) quality of export fruit ( $\left(7^{\text {st }}\right.$ and $2^{\text {nd }}$ ) with 3 repetitions. The variables studied were based on 20 clusters. From the results obtained, it follows that the physical and chemical quality of the fruit is influenced by the harvest season, since they grew and developed in different environmental conditions. In general, $82 \%$ of the production is destined for export, since it meets the quality standards.
\end{abstract}

\section{Keywords}

Organic banana; physical quality; chemical quality, post-harvest losses, fruit discards.

\section{Introducción}

La superficie mundial de banano cultivado en el mundo es de 6719720 ha, y se cosechan 93 390721 t (USAID, 2011). La variedad de mayor producción es el tipo Cavendish (47 \%); seguido del banano de tierras altas (ABB) con el $24 \%$; el plátano (AAB) con $17 \%$, y Gross Michel con el $12 \%$ (FAO, 2004). 
Ecuador es el primer exportador de banano en el mundo (Asociación de Exportadores de Banano del Ecuador [AEBE], 2016), con una superficie plantada de 196673 ha y una producción de 317437040 cajas (18.14 kg caja-1), de las cuales en el trópico se produce el 89 \%; en la zona baja de la Sierra, $10 \%$, y en el Oriente, 1\%. Existe un incremento en la producción del $6.5 \%$ en comparación con el 2014 (AEBE, 2016). Las principales exportaciones fueron enviadas a Rusia 20.3 \%; Estados Unidos, $15.5 \%$, y Alemania, 11.5 \% (FAO, 2018). El cultivo de banano representa el $12.39 \%$ de la superficie total agrícola del Ecuador (INEC, 2015). La industria de banano aporta el $2 \%$ del PIB nacional y el $26 \%$ al PIB agrícola (INEC, 2009). La industria bananera genera 4 plazas de empleo directo por ha y 2.5 millones de empleos directos e indirectos (Proecuador, 2016).

América Latina es el principal exportador de banano orgánico con 24.2 millones de cajas en el año 2009, lo cual representa el $3 \%$ del banano convencional (Soto, 2011). La superficie de banano orgánico en Ecuador es de 19000 ha (Proecuador, 2016) con una producción de 360 000 t año-1 $^{-1}$ Estrella, 2004). El principal destino de banano orgánico es Estados Unidos, Europa y Nueva Zelanda (Soto, 2011 y United Nations Conference on Trade and Development, 2014).

En cuanto a las pérdidas poscosecha de banano, estas van del $10 \%$ al $80 \%$. Las pérdidas son causadas por daños mecánicos, plagas, maduración prematura, deformidades, manipuleo, entre otros (FAO, 2005). La fruta que no es apta para la exportación, debido a los estándares de alta calidad exigidos por los importadores, es utilizada en la agroindustria, para alimentos de animales o fruta fresca para el mercado nacional (FONTAGRO, 2006).

Entre los insectos plagas, los picudos (Cosmopolites sordidus, Metamasius hemipterus, Metamasius hebetatus) perjudican a la planta, puesto que tienen hábitos nocturnos; se alimentan del seudotallo, lo que afecta el rendimiento (60\%) y causa el volcamiento de la planta. Los nematodos (Radopholus similis) son plagas microscópicas del suelo que dañan las raíces (Alarcón y Jiménez, 2012) y causan clorosis a la planta. Asimismo, los trips (Chaetanaphothrips signipennis, Frankliniella brevicaulis) producen manchas de color rojizo (mancha roja) en la corteza del fruto (Ministerio de Agricultura y Riego, 2013). La cochinilla (Pseudococcidae) afecta a la planta durante el crecimiento vegetativo, floración, fructificación, y después de la cosecha, lo que impide la exportación (MAGAP y Agrocalidad, 2015). Para prevenir el daño en forma biológica, se pueden utilizar hormigas depredadoras (Pheidole megacephala), nematodos y hongos entomopatógenos, formulaciones de aceites, entre otros (Carballo, 2001 y Alarcón y Jiménez, 2012).

Una de las enfermedades prevalentes en el cultivo de banano es la sigatoka negra (Mycosphaerella fijiensis). Esta enfermedad, que se presenta durante todo el ciclo del cultivo, perjudica a las hojas y produce manchas alargadas que matan el tejido foliar de la planta (Alarcón y Jiménez, 2012). Para evitar la propagación de la sigatoka negra en cultivo orgánicos, se debe prevenir empleando prácticas culturales y utilizando bioproductos y variedades resistentes (Carballo, 2001).

El banano es una fruta climatérica (Knee, 2008). Una vez cosechado el fruto, se procede a desinfectarlo para evitar el ataque de las plagas (Agrocalidad, 2014). La selección permite separar los frutos buenos de los dañados, tales como los deformes, con daños físicos, infectados de plagas (Sena et al., 2004). La clasificación del fruto se hace de acuerdo con las características físicas como el tamaño (longitud y diámetro), el grado de madurez y la categoría (extra, primera y segunda), entre otras.

La calidad física se sustenta en la apariencia de la fruta (tamaño, forma, color, brillo, firmeza, ausencia de defectos y deterioro). La calidad nutricional se determina por la presencia de minerales, vitaminas, fibra alimenticia, pH, sólidos solubles totales y acidez (Millán y Ciro, 2012, 
y Knee, 2008). Con este antecedente, el estudio tiene el objetivo de determinar la calidad del fruto con base en las características físicas como tamaño, masa, diámetro, firmeza, químicas como el pH, acidez y sólidos solubles totales, y cuantificar las pérdidas poscosecha del banano orgánico según las épocas de cosecha. La hipótesis planteada es que las condiciones ambientales influyen en la calidad del fruto de banano orgánico.

\section{Metodología}

El estudio se realizó en una empresa productora de banano orgánico ubicada a 79 48' 33.55" W de longitud y $1^{\circ} 37^{\prime} 26.36^{\prime \prime}$ S de latitud, del cantón de Vinces, provincia de Los Ríos. La temperatura promedio fue de $26{ }^{\circ} \mathrm{C}$, con una precipitación acumulada que fluctuó entre 1500-2500 mm y humedad relativa de $98 \%$.

Para el estudio se utilizó un diseño de bloques completamente al azar (DBCA) en arreglo factorial $7 \times 2$. Los factores fueron los siguientes: 1. épocas de cosecha (febrero-agosto), y 2. calidad de fruta de exportación (primera y segunda) con 3 repeticiones. La comparación de medias se hizo mediante la prueba de Tukey $(P \leq 0.05)$.

Las variables estudiadas fueron las siguientes:

Masa del fruto (g), para esta se empleó una balanza analítica; la muestra fue de 20 frutos;

El tamaño del fruto (cm) se determinó por medio de un calibrador y una cinta métrica.

El pH se registró con potenciómetro. Se licuaron $30 \mathrm{~g}$ de pulpa de fruta y $90 \mathrm{ml}$ de agua destilada y se filtró en un colador. La lectura fue directa;

La acidez titulable con base en el ácido málico, para esto se preparó una solución compuesta de $25 \mathrm{ml}$ del jugo de la fruta. Esta solución se tituló con $\mathrm{NaOH}$ al $0.1 \mathrm{~N}$ hasta llegar a un pH de 8.1; se determinó la cantidad $(\mathrm{ml})$ de $\mathrm{NaOH}$ y se procedió a calcular según el protocolo de Dadzi (1997);

Los sólidos solubles totales (SST) se obtuvieron con un refractómetro digital, para lo cual se puso unas gotas del jugo de la fruta en el prisma del refractómetro; la lectura fue de forma directa;

La firmeza del fruto se midió con un penetrómetro; se consideró una muestra de 20 frutos seleccionados al azar de cada tratamiento, y

La pérdida de la fruta durante el manejo de poscosecha se consideró desde que los racimos llegaron a la empacadora hasta que fueron puestos en las cajas. La unidad experimental fue de 20 racimos. Se registró la cantidad $(\mathrm{kg})$ total de la fruta al ingreso a la empacadora y los pesos de las pérdidas, debido a los daños físicos (manipuleo, deformes), fisiológicos y de plagas.

En la Tabla 1, se presentan las diferentes condiciones climáticas que se registraron durante el crecimiento de los racimos desde la floración hasta la cosecha de la fruta, en la finca ubicada en el cantón de Vinces, provincia de Los Ríos. Como se puede apreciar, hubo una gran variación en la precipitación acumulada, con una fluctuación entre 565 hasta 1882 mm. Igual ocurrió en la acumulación de las horas luz, con una variación de 260 a 444 h. No obstante, la temperatura y la humedad relativa promedio tuvieron una fluctuación mínima. Esta información es muy importante, ya que las condiciones climáticas influenciaron en el crecimiento y desarrollo del racimo, así como también en la presencia de las plagas. Estas consideraciones se deben tener en cuenta en el diseño del manejo integrado de plagas, más aún en la producción orgánica. 
Tabla 1. Condiciones climáticas durante el desarrollo y crecimiento de los racimos cosechados en el trópico ecuatoriano

\begin{tabular}{|l|l|l|l|l|}
\hline \multicolumn{1}{|c|}{ Meses/2016 } & \multicolumn{1}{|c|}{$\begin{array}{c}\text { Temperatura } \\
\text { promedio }\left({ }^{\circ} \mathbf{C}\right)\end{array}$} & \multicolumn{1}{|c|}{$\begin{array}{c}\text { Precipitación } \\
\text { acumulada }(\mathbf{m m})\end{array}$} & $\begin{array}{c}\text { Humedad relativa } \\
\text { promedio }(\%)\end{array}$ & \multicolumn{1}{|c|}{$\begin{array}{c}\text { Horas luz } \\
\text { acumuladas }\end{array}$} \\
\hline Enero & 21.8 & 931.4 & 83.0 & 260.2 \\
\hline Febrero & 22.0 & 1229.8 & 84.5 & 278.6 \\
\hline Marzo & 22.4 & 1674.4 & 85.8 & 327.6 \\
\hline Abril & 22.4 & 1882.9 & 86.3 & 375.7 \\
\hline Mayo & 22.3 & 1566.4 & 85.3 & 444.2 \\
\hline Junio & 21.8 & 1188.9 & 84.0 & 438.9 \\
\hline Julio & 21.2 & 565.4 & 83.5 & 421.7 \\
\hline Agosto & 20.0 & 126.2 & 81.5 & 313.2 \\
\hline
\end{tabular}

Adaptada de INAMHI (2016)

\section{Resultados y discusión}

\subsection{Caracteristicas físicas del fruto}

En general, el estudio permitió determinar el efecto de las condiciones ambientales durante el período de producción en las características físicas del fruto de banano. Respecto a la calidad física de la fruta, se encontraron diferencias para masa (g), longitud y diámetro (mm) del fruto, características determinantes a la hora de seleccionar la fruta para exportación.

La mayor masa del fruto se registró en el mes de abril, seguido del mes de marzo con 200 y $180 \mathrm{~g}$ respectivamente; mientras que en el mes de febrero y julio los frutos fueron los de menor masa con 160 y $150 \mathrm{~g}$ respectivamente (ver Tabla 2). Esta misma tendencia se presentó para la longitud $(24.22$ y $23.52 \mathrm{~cm})$ y diámetro del fruto $(3.67$ y $3.48 \mathrm{~cm})$ del fruto respectivamente. Por su parte Martínez, Blanco, Hernández, Manzanilla, Pérez, Pargas y Marín (2009), señalan que el tamaño del racimo de banano está relacionado con la densidad de plantación, ya que a menor densidad los racimos y los frutos son de mayor tamaño, debido a la menor competencia por nutrientes y agua que tiene la planta. La firmeza de los frutos también fue afectada por las condiciones climáticas que prevalecieron durante el período de desarrollo; los frutos cosechados en el mes de febrero tuvieron mayor firmeza (9.16 lbf) que los cosechados en junio (6.38 lbf) (ver Tabla 2).

Tabla 2. Promedios de cuatro características físicas del fruto de banano orgánico cosechados en diferentes épocas

\begin{tabular}{|l|l|l|l|l|}
\hline \multicolumn{1}{|c|}{ Cosecha } & \multicolumn{1}{|c|}{$\begin{array}{c}\text { Masa fruto } \\
(\mathbf{g})\end{array}$} & \multicolumn{1}{|c|}{$\begin{array}{c}\text { Longitud de fruta } \\
(\mathbf{c m})\end{array}$} & \multicolumn{1}{c|}{$\begin{array}{c}\text { Diámetro del fruto } \\
(\mathbf{c m})\end{array}$} & \multicolumn{1}{|c|}{$\begin{array}{c}\text { Firmeza del fruto } \\
\text { (Ibf) }\end{array}$} \\
\hline Febrero & $160 \pm 0.00 \mathrm{c}$ & $21.05 \pm 0.10 \mathrm{c}$ & $3.36 \pm 0.06 \mathrm{~b}$ & $9.16 \pm 0.28 \mathrm{a}$ \\
\hline Marzo & $180 \pm 0.00 \mathrm{ab}$ & $23.52 \pm 0.57 \mathrm{ab}$ & $3.48 \pm 0.03 \mathrm{ab}$ & $8.90 \pm 0.23 \mathrm{ab}$ \\
\hline Abril & $200 \pm 0.01 \mathrm{a}$ & $24.22 \pm 0.45 \mathrm{a}$ & $3.67 \pm 0.07 \mathrm{a}$ & $8.69 \pm 0.10 \mathrm{abc}$ \\
\hline Mayo & $170 \pm 0.02 \mathrm{bc}$ & $22.06 \pm 0.47 \mathrm{bc}$ & $3.44 \pm 0.19 \mathrm{ab}$ & $8.12 \pm 0.17 \mathrm{c}$ \\
\hline Junio & $160 \pm 0.00 \mathrm{bc}$ & $21.57 \pm 0.21 \mathrm{c}$ & $3.40 \pm 0.08 \mathrm{~b}$ & $6.38 \pm 0.33 \mathrm{~d}$ \\
\hline Julio & $150 \pm 0.01 \mathrm{c}$ & $20.91 \pm 0.23 \mathrm{c}$ & $3.36 \pm 0.05 \mathrm{~b}$ & $9.03 \pm 0.37 \mathrm{a}$ \\
\hline Agosto & $160 \pm 0.01 \mathrm{bc}$ & $22.44 \pm 1.38 \mathrm{bc}$ & $3.43 \pm 0.10 \mathrm{~b}$ & $8.19 \pm 0.39 \mathrm{bc}$ \\
\hline
\end{tabular}

Promedios seguidos de la misma letra son estadísticamente iguales con Tukey (5\%) 
Respecto a la calidad de exportación del fruto (ver Tabla 3), se observó que los bananos valorados como de primera tienen una masa promedio de $180 \mathrm{~g}$, mientras que los de segunda, 160 g. Adicionalmente, otros parámetros tomados en cuenta para que la fruta sea considerada de segunda son la presencia de mancha roja, daños físicos y cicatrices hasta un $1 \%$. Respecto a longitud y diámetro de la fruta de exportación, primera fueron $1 \mathrm{~cm}$ más largos que los de segunda (ver Tabla 3). Igual tendencia se encontró en el diámetro, es decir, mayor diámetro tuvieron los frutos de primera $(3.53 \mathrm{~cm})$ frente a los de segunda $(3.37 \mathrm{~cm})$.

Tabla 3. Promedios de las características físicas del fruto de banano orgánico en función de la calidad de la exportación

\begin{tabular}{|l|l|l|l|}
\hline \multicolumn{1}{|c|}{ Calidad exportación } & \multicolumn{1}{|c|}{$\begin{array}{c}\text { Peso del fruto } \\
\mathbf{( g )}\end{array}$} & \multicolumn{1}{|c|}{$\begin{array}{c}\text { Longitud del fruto } \\
\text { (cm) }\end{array}$} & \multicolumn{1}{|c|}{$\begin{array}{c}\text { Diámetro del fruto } \\
\text { (cm) }\end{array}$} \\
\hline primera & $180 \pm 0.00 \mathrm{a}$ & $22.82 \pm 0.15 \mathrm{a}$ & $3.53 \pm 0.04 \mathrm{a}$ \\
\hline segunda & $160 \pm 0.01 \mathrm{~b}$ & $21.69 \pm 0.43 \mathrm{~b}$ & $3.37 \pm 0.06 \mathrm{~b}$ \\
\hline
\end{tabular}

Promedios seguidos de las mismas letras son iguales estadísticamente con Tukey $(P \leq 0.05)$

Con base en estos resultados, se puede inferir que las diferencias en la calidad física de los frutos en gran medida se deben al efecto de las condiciones climáticas que prevalecieron durante el período de crecimiento y desarrollo del fruto. Como se puede ver en la Tabla 1, la precipitación y las horas luz fueron las que más incidieron; estos resultados concuerdan con lo reportado por Galán y Robinson (2013). Por su parte Yela, Boza, Baquedano, Fierro, Rivas y Quiñonez (2016) señalan que las variaciones del clima como la temperatura, la presencia de vientos y la distribución de las lluvias afectan directamente a la producción de la fruta. Además, Knee (2008) asegura que el estrés hídrico y el déficit de nutrientes inciden en el tamaño de la fruta. Es relevante indicar que la firmeza de los frutos es crucial para el manejo poscosecha, puesto que a menor firmeza los frutos, son más sensible a daños por manipulación (Campuzano, Cornejo, Ruiz y Peralta, 2010), por lo tanto, se debe cosechar el fruto en el punto óptimo considerando el destino y mercado (Ciro, Montoya y Millán, 2005).

\subsection{Características químicas del fruto}

Los análisis estadísticos demostraron que no hubo efecto de la época de cosecha en los SST y el pH del fruto, pero sí en la acidez, si se considerael ácido málico. Los frutos cosechados en el mes de marzo presentan mayor acidez (0.53) que los de febrero (0.42) (ver Tabla 4). La acidez titulable es un indicador de la calidad de la fruta, debido a que presenta un equilibrio entre el contenido de azúcares $\left(<5^{\circ} \mathrm{Brix}\right.$ ) y la acidez $(\approx 0.5 \%)$, los cuales se incrementan conforme madura la fruta (Barrera, Arrazola y Cayón, 2010). Se conoce que la acidez influye en el sabor y puede ser considerada un indicador de madurez, tal como lo reportan Dadzie y Orchard (1997), lo cual fue corroborado por Campuzano et al (2010). 
Tabla 4. Promedios de la acidez titulable de los frutos de banano orgánico cosechados en diferentes fechas

\begin{tabular}{|l|l|}
\hline \multicolumn{1}{|c|}{ Cosechas } & \multicolumn{1}{c|}{ Acidez titulable (\%) } \\
\hline Febrero & $0.42 \pm 0.01 \mathrm{c}$ \\
\hline Marzo & $0.53 \pm 0.03 \mathrm{a}$ \\
\hline Abril & $0.51 \pm 0.05 \mathrm{a}$ \\
\hline Mayo & $0.51 \pm 0.02 \mathrm{a}$ \\
\hline Junio & $0.51 \pm 0.01 \mathrm{a}$ \\
\hline Julio & $0.50 \pm 0.05 \mathrm{ab}$ \\
\hline
\end{tabular}

Promedios seguidos de las mismas letras son iguales estadísticamente con Tukey $(P \leq 0.05)$

\subsection{Calidad de la fruta}

La cantidad de fruta de banano exportado de la primera categoría es mayor en los primeros meses del año (febrero-junio) comparada con los últimos meses del período del estudio (agosto), en el que se observa una fluctuación entre el 70 y 90 \% de la producción. Mientras que con la fruta de segunda categoría de exportación sucede lo contrario, pues presenta una fluctuación entre el 0.4 y $12.7 \%$ (ver Tabla 5). Las principales causas del descarte de la fruta cosechada es la manipulación durante la cosecha y poscosecha (6.6 \%), frutos con mancha roja (16.5 \%) causado por los trips y dedos laterales abiertos de la mano (16.2\%). El mayor descarte de la fruta de banano se presentó en el mes de marzo (23.5 \%). En general, el descarte de la fruta esta dado por la calidad, la cual se produce en función del componente genético, del manejo agronómico ("Colombia brilla", 2010) y también de las condiciones ambientales (Vásquez-Castillo et al., 2016).

Tabla 5. Fruta de exportación (\%) y descarte (\%) de banano orgánico en función de los estándares de calidad del mercado

\begin{tabular}{|l|l|l|l|l|l|l|l|l|l|l|l|}
\hline \multirow{2}{*}{$\begin{array}{c}\text { Fecha } \\
\text { Cosecha }\end{array}$} & \multicolumn{2}{|c|}{ Exportación (\%) } & \multicolumn{7}{c|}{ Descarte (\%) } \\
\cline { 2 - 12 } & primera & segunda & $\begin{array}{c}\text { Dedo late- } \\
\text { ral abierto }\end{array}$ & $\begin{array}{c}\text { Mancha } \\
\text { roja }\end{array}$ & manipuleo & $\begin{array}{c}\text { Dedos } \\
\text { deformes }\end{array}$ & $\begin{array}{c}\text { Dedos } \\
\text { cortos }\end{array}$ & $\begin{array}{c}\text { Madurez } \\
\text { inadecuada }\end{array}$ & $\begin{array}{c}\text { Daño } \\
\text { plagas }\end{array}$ & Total \\
\hline Febrero & 88.9 & 1.4 & 1.4 & 0.9 & 1.9 & 1.3 & 1 & 0 & 0 & 6.5 \\
\hline Marzo & 76 & 0.4 & 2.3 & 12.5 & 4.5 & 0.5 & 0 & 3.1 & 0.7 & 23.6 \\
\hline Abril & 89.5 & 4 & 4.9 & 1.5 & 2.8 & 0.9 & 1.3 & 0.1 & 0 & 11.5 \\
\hline Mayo & 87.7 & 4.9 & 1.5 & 0 & 3.2 & 1.5 & 3.9 & 1 & 1.1 & 12.2 \\
\hline Junio & 85.6 & 1.7 & 2.0 & 0.7 & 2.5 & 1.1 & 2.5 & 0 & 0 & 8.8 \\
\hline Julio & 80.4 & 3.7 & 1.9 & 0 & 1.2 & 0.9 & 3.8 & 0.6 & 0.8 & 9.2 \\
\hline Agosto & 70.8 & 12.7 & 2.2 & 0.9 & 0.5 & 0.2 & 0.4 & 2.3 & 0.1 & 6.6 \\
\hline
\end{tabular}

\section{Cosecha y manejo de la poscosecha}

Para la cosecha, se verificó el grado de madurez de los frutos, para lo cual se consideró el color de la cinta que tienen los racimos en el campo. También se midió el calibre de los frutos localiza- 
dos en la parte media de la última mano del racimo;el calibre debe estar entre 38 y $48 \mathrm{~mm}$. Una vez cosechados, se transportó los racimos a la empacadora por medio del cable vía.

En la recepción del racimo se registró el peso de los dos últimos racimos de cada lote y se identificó el número del lote. Se inspeccionó el largo del fruto (> a $21 \mathrm{~cm}$ ), el color de la almendra (pulpa), la cual no debe tener manchas amarillas. Posteriormente, se procedió al lavado y control de cochinilla antes del desmane. Las manos se ponen en una tina con agua para eliminar el látex. Luego, los frutos fueron clasificados y seleccionados con base en los requerimientos del mercado internacional. La fruta se clasifica según el tamaño y el grado de madurez; cada gajo debe tener entre 5 y 8 dedos. Se lavó nuevamente con cloro, para eliminar cualquier residuo que haya quedado después del primer lavado. A continuación, se pusieron los gajos de banano en las bandejas para llenar una caja de fruta $(18.86 \mathrm{~kg})$. El curado de la corona se hizo para sellar el corte utilizando un producto aprobado por la certificadora y también con parafilm. Luego, las cajas se etiquetaron y empacaron, $y$, finalmente, fueron embarcadas en los contenedores climatizados a una temperatura entre 13.3 a $14.5^{\circ} \mathrm{C}$, y a una humedad relativa controlada entre 85 a $95 \%$ para preservar la fruta. Este proceso concuerda con el manejo poscosecha reportado por Riofrío (2003). En la Figura 1, se detalla el proceso desde la cosecha hasta la poscosecha, mediante un flujograma.

Figura 1. Flujograma de la cosecha y manejo poscosecha de la producción de banano orgánico

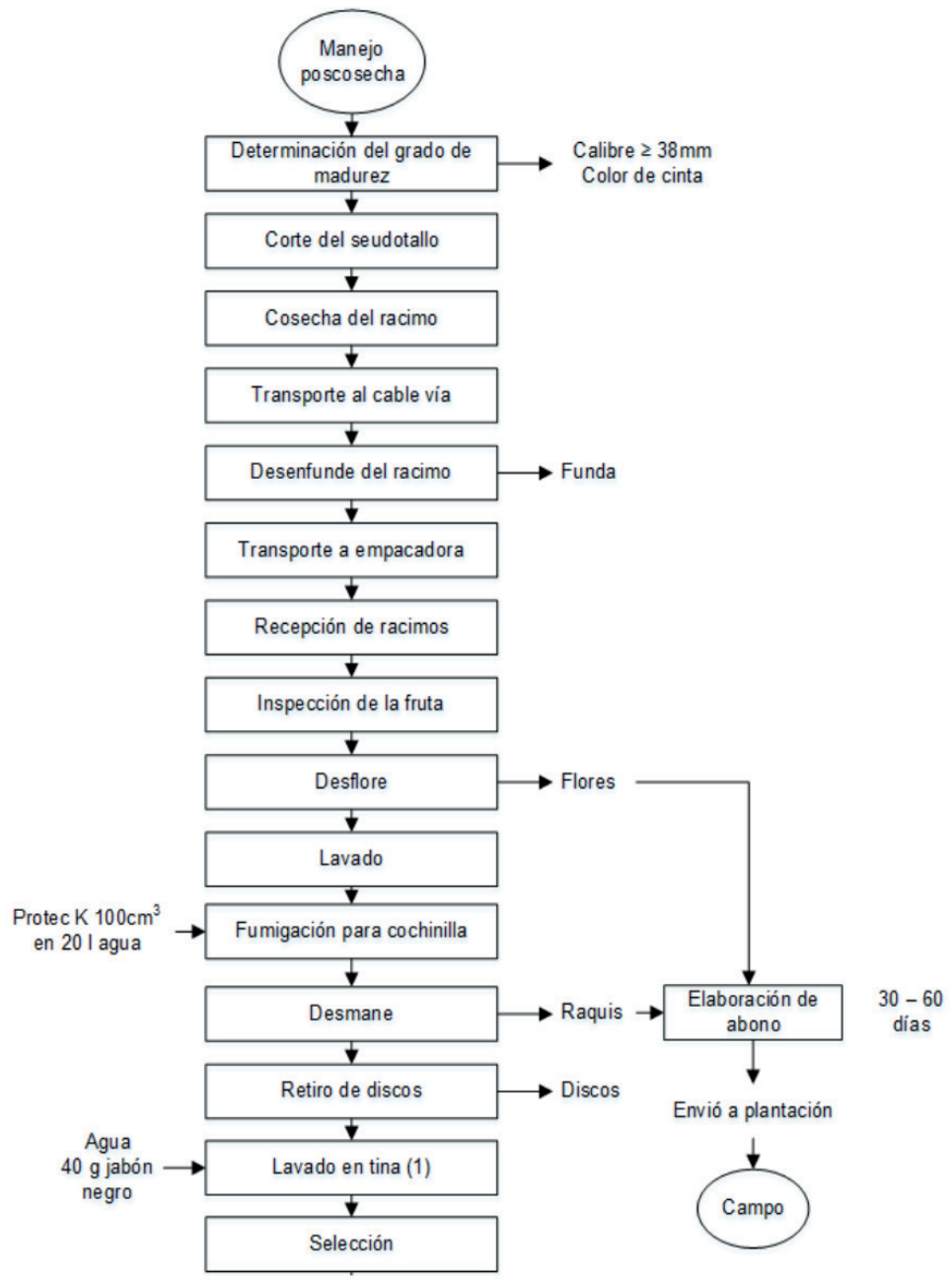




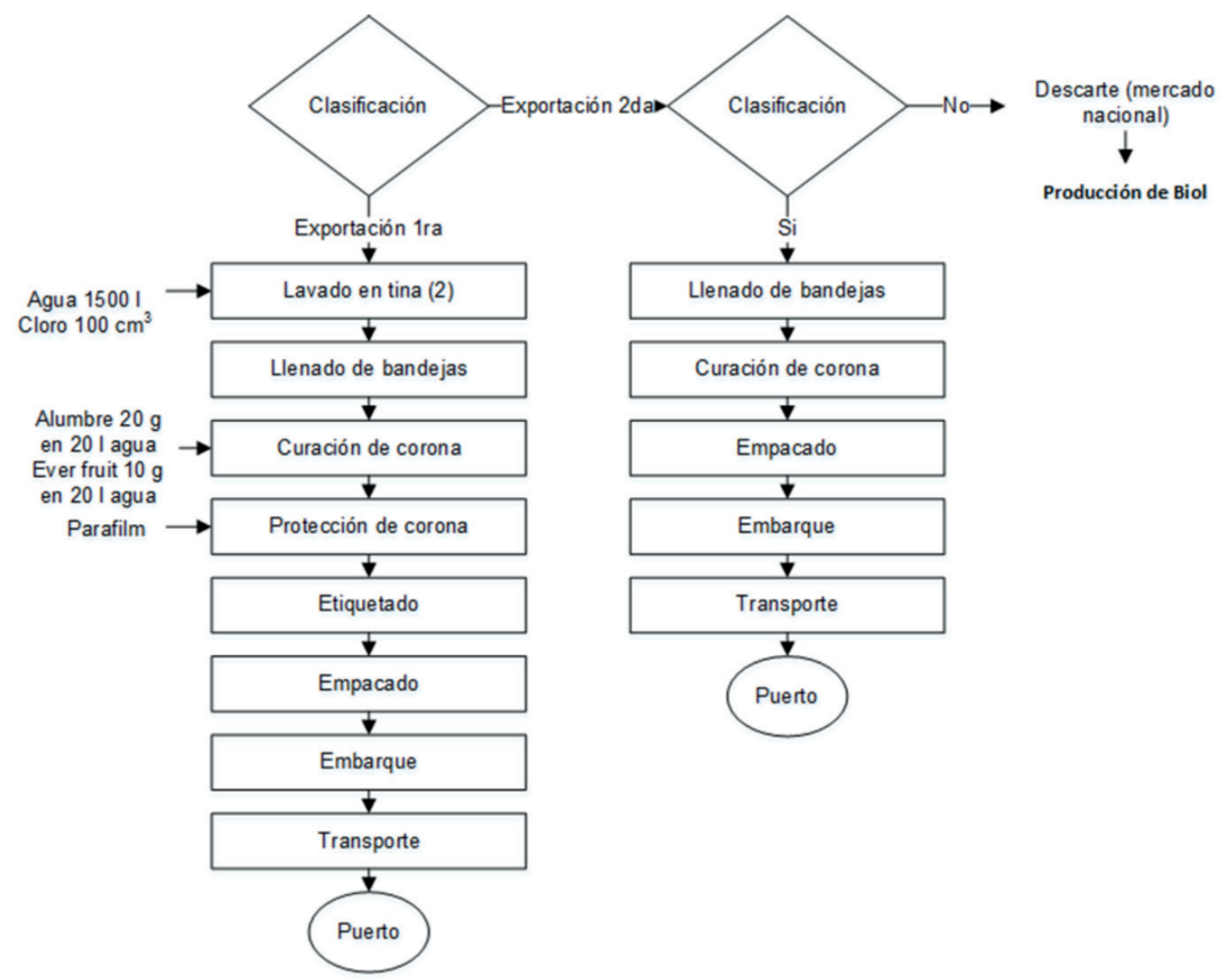

\section{Conclusiones y recomendaciones}

La calidad física del fruto de banano orgánico está influenciada por la época de cosecha, debido a que tuvieron diferentes condiciones ambientales durante el crecimiento y desarrollo. La acidez titulable fue la única característica química que estuvo influenciada por la época de cosecha. Se determinó que más del 80 \% de la cosecha se destina a la exportación de fruta de primera categoría.

Con el fin de que el porcentaje de fruta para la exportación sea consistente, se debe llevar a cabo un estudio para determinar los puntos críticos del sistema de producción y encontrar alternativas tecnológicas para estandarizar los procesos.

\section{Agradecimiento}

Los autores agradecemos al Dr. Trevor Jackson, investigador de AgResearch, por el aporte técnico. Al gobierno de Nueva Zelandia (MFAT New Zealand) y a la Escuela Politécnica Nacional por el apoyo económico. También agradecemos a los organizadores del II Simposio Internacional de Producción Integrada de Frutas 2019 por permitirnos difundir los resultados de este estudio. 


\section{Bibliografía}

Asociación de Exportadores de Banano del Ecuador. (2016). Exportaciones mensuales de banano. Recuperado de http://www.aebe.com.ec/wp-content/uploads/2018/06/AE_EstadisticasPublicas_Mar18.pdf

Agrocalidad. (2014). Buenas prácticas agrícolas para el banano. Recuperado de http://www.agrocalidad. gob.ec/wp-content/uploads/pdf/inocuidad/BANANO/banano.pdf

Alarcón, J. y Jiménez, Y. (2012). Manejo fitosanitario del cultivo del plátano. Recuperado de http://www. fao.org/fileadmin/templates/banana/documents/Docs_Resources_ 2015/TR4/cartilla-platano-ICA-final-BAJA.pdf

Barrera, J.; Arrazola G., y Cayón, E. (2010). Caracterización fisicoquímica y fisiológica del proceso de maduración de plátano Hartón (musa AAB Simmonds) en dos sistemas de producción. Acta Agronómica, 59(1), 20-29.

Campuzano, A.; Cornejo, F.; Ruiz, O., Peralta, E. (2010). Efecto del tipo de producción de banano cavendish en su comportamiento poscosecha. Revista Tecnológica ESPOL-RTE, 23(2), 41-48.

Carballo, M. (2001). Opciones para el manejo del picudo negro del plátano. Manejo integrado de plagas, 59, i-iv Recuperado el de 2016. https://www.semanticscholar.org/paper/Opciones-para-elmanejo-del-picudo-negro-del-Carballo/2ddb5d25580a00fb94a3caaca15e1a029c4ee083\#p aper-header

Ciro, H.; Montoya, M., y Millán, L. (2005). Caracterización de propiedades mecánicas del banano (Cavendish Valery). Revista de la Facultad Nacional de Agronomía Medellín, 58(2), 2975-2988.

Colombia brilla con pitahaya amarilla. (julio de 2010). Sembramos, edición 13, 14.

Dadzie, B. y Orchard, J. (1997). Evaluación rutinaria poscosecha de híbridos de banana y plátanos: Criterios y métodos. Guías técnicas INIBAP 2. Montpellier, Francia: INIBAP.

Estrella, l. (2004). Agricultura orgánica Ecuador. Recuperado de http://docplayer.es/15385542-Agricultura-organica-ecuador-septiembre-2004-liggia-estrella-corpei.html

FAO. (2005). Pérdidas en la manipulación después de la cosecha. Recuperado de ftp://ftp.fao.org/docrep/fao/meeting/009/j 5778s.pdf

FAO. (2004). La economía mundial del banano 1985-2002. Publicación preparada por Arias, P., Dankers, C., Liu, P., et al. Recuperado de http://www.fao.org/3/y5102s/y5102s00.htm

FAO. (2018). Situación del mercado del banano: Resultados preliminares relativos a 2017. Recuperado de http://www.fao.org/fileadmin/templates/est/COMM_MARKETS_MONITORING/Bananas/Documents/Spanish_December_2017_update.pdf

FONTAGRO. (2006). Informe Fontagro. Recuperado de http://s1. fontagro.org/sites/default/files/pro p_06_05.pdf

Galán, V. y Robinson, J. (2013). Fisiología, clima y producción de banano. XX Reunião Internacional da Associação para a Cooperação em Pesquisa e Desenvolvimento Integral das Musáceas (Bananas e Plátanos). 43-57

INEC. (2011). Reporte estadístico del sector agropecuario. Agosto 2011. Recuperado de https://www. ecuadorencifras.gob.ec/wp-content/descargas/Presentaciones/espac_2010.pdf

INEC. (2015). Encuesta de superficie y producción agropecuaria continua ESPAC 2016. Recuperado de https://www.ecuadorencifras.gob.ec/documentos/web-inec/Estadisticas_agropecuarias/espac/espac-2016/Informe\%20ejecutivo\%20ESPAC_2016.pdf

Knee, M. (2008). Bases biológicas de la calidad de la fruta. Zaragoza, España: Acribia. do.pdf

Martínez, G.; Blanco, G.; Hernández, J.; Manzanilla, E; Pérez, A.; Pargas, y Marín, C (2009). Comportamiento del plátano (Musa AAB Subgrupo plátano, cv. Hartón Gigante) sembrado a diferentes densidades de siembra en el Estado Yaracuy, Venezuela. Revista Científica UDO Agrícola 9(1): 259-267. 
Millán, L. y Ciro, H. (2012). Caracterización mecánica y físico-química del banano tipo exportación (Cavendish valery). Corporación Universitaria Lasallista. Recuperado de http://repository.lasallista. edu.co/dspace/bitstream/10567/136/1/10.\%20163-192.pdf

MAGAP y Agrocalidad. (2015). Guía fitosanitaria de campo cultivo de banano. Recuperado de http://www. agrocalidad.gob.ec/wp-content/uploads/2015/04/guia-de-campo-banano.pdf

Ministerio de Agricultura y Riego. (2013). Manejo integrado de plagas y enfermedades en banano orgánico. Recuperado de http://agroaldia.minagri.gob.pe/biblioteca/download/pdf/manuales-boletines/banano/banano_integrado.pdf

Proecuador. (2016). Análisis sectorial: Banana 2016. Recuperado de http://www.proecuador.gob.ec/wpcontent/uploads/2016/09/PROEC_AS2 016_BANANO.pdf

Riofrío, J. (2003). Manejo post-cosecha del banano y plátano (p. 168). Administración del control de calidad en productos hortifrutícolas T. iii. Guayaquil, Ecuador.

Sena; Secretaría de Agricultura del Meta; Corpometa, Asohofrucol. (2004). Plátano, su cosecha y poscosecha en la cadena agroindustrial. Recuperado de http://www.asohofrucol.com.co/archivos/ biblioteca/biblioteca_26_platano.pdf

Soto, M. (2011). Situación y avances tecnológicos en la producción bananera mundial. Revista Brasileira de Fruticultura 33(1),13-28. doi.org/10.1590/S0100-29452011000500004

United Nations Conference on Trade and Development. (2014). Estudio base para la revisión de la política de exportación de productos verdes del Ecuador. Recuperado de http://unctad.org/en/ PublicationsLibrary/webditcted2014d4_es.pdf

USAID. (2011). Banana: Análisis de la cadena de valor en el departamento de San Pedro. Publicación preparada por Fretes., F y Martínez, M. Recuperado de https://www.usaid.gov/sites/default/files/ documents/1862/banana.pdf

Vásquez-Castillo, W.; Aguilar, K.; Vilaplana, et al. (2016). Calidad del fruto y pérdidas poscosecha de pitahaya amarilla (Selenicereus megalanthus Haw) en Ecuador. Agronomia Colombiana, 34(1Supl.), 1081-1083. doi: 10.15446/agron.colomb.v34n1supl.58279

Yela, P.; Boza, J.; Baquedano, L.; Fierro; Rivas, K., y Quiñonez (2016). Efectos del cambio climático en la producción agrícola del banano en el cantón Valencia. Revista Caribeña de Ciencias Sociales, en línea. Recuperado el de http://www.eumed.net/rev/caribe/ 2016/09/banano.html 
